\title{
5-(1H-1,2,3-Triazol-5-yl)isophthalic Acid: A Versatile Ligand for the Synthesis of New Supramolecular Metallogels
}

Marleen Häring, ${ }^{\dagger}$ Sujay Kumar Nandi, ${ }^{\ddagger}$ Julio Rodríguez-López, $^{\S}$ Debasish Haldar, ${ }^{\ddagger}$ Víctor S. Martín, $^{\S}$ Antonio Diego Lozano-Gorrín, ${ }^{\|, \perp}$ César Saldías, ${ }^{\#}$ and David Díaz Díaz ${ }^{*, \dagger, \text { II }}$

${ }^{\dagger}$ Institut für Organische Chemie, Universität Regensburg, Universitätsstr. 31, Regensburg D-93053, Germany

${ }^{\ddagger}$ Department of Chemical Sciences, Indian Institute of Science Education and Research Kolkata, Mohanpur 741246, West Bengal, India

${ }^{\S}$ Instituto Universitario de Bio-Orgánica “Antonio González” (CIBICAN), “Síntesis Orgánica Sostenible, Unidad Asociada al CSIC”, Departamento de Química Orgánica, Universidad de La Laguna, Francisco Sánchez 2, La Laguna 38206, Tenerife, Spain

"Departamento de Química, Universidad de La Laguna, La Laguna 38206, Tenerife, Spain

${ }^{\perp}$ Instituto Universitario de Materiales y Nanotecnología (IMN), Universidad de La Laguna, La Laguna 38200, Tenerife, Spain

\#Departamento de Química Física, Facultad de Química y de Farmacia, Pontificia Universidad Católica de Chile, Macul, Santiago 7820436, Chile

II Instituto de Productos Naturales y Agrobiología del CSIC, Avda. Astrofísico Francisco Sánchez 3, La Laguna 38206, Tenerife, Spain

\section{Supporting Information}

ABSTRACT: The gelation ability of 5-(1H-1,2,3-triazol-5-yl)isophthalic acid (clickTIA) in the presence of different metal acetates has been studied in different solvents and ligand/metal ratios. This manuscript is focused on the metallogel obtained from the combination of click-TIA and copper(II) acetate, which has been used as a model system in terms of characterization and gelation studies. Sonication treatment of the initial mixture of compounds and the nature of the counter anion were found to be critical factors for the supramolecular assembly of the metal/click-TIA complexes and, hence, for the formation of stable and homogeneous metallogels. The gel materials have been characterized with a variety of techniques including infrared, rheology, UV-vis spectroscopy, powder X-ray diffraction, and scanning electron microscopy.

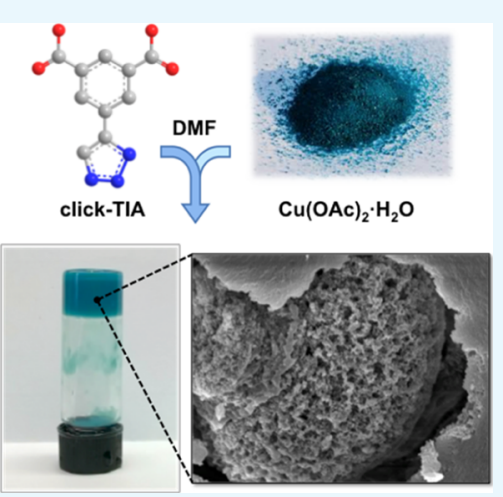

\section{INTRODUCTION}

Supramolecular gels have gained great attention because of their potential applications in diverse fields including, among others, biomedicines, ${ }^{1-4}$ sensors, ${ }^{5,6}$ catalysis, ${ }^{7}$ environmental remediation, ${ }^{8-10}$ and material synthesis. ${ }^{11-13}$ These viscoelastic materials are made by self-assembly of either low-molecular weight (LMW) molecules or polymers (so-called gelators), via noncovalent interactions (e.g., hydrogen-bonding, van der Waals, $\pi-\pi$ stacking, charge-transfer, coordination interactions, etc). ${ }^{14-19}$ The solid-like appearance of these materials and their rheological properties are the result of the immobilization of the liquid phase (major component) in the interstices of an entangled self-assembled solid matrix, usually by capillary forces and surface tension phenomena. ${ }^{20-25}$ Metallogels, containing often discrete metal coordination complexes of LMW ligands or well-defined coordination polymers, are promising materials for numerous applications in materials science because of their redox, electronic, magnetic, and optical properties, providing a variety of stimuli-responsive "smart" materials and catalytic gels. ${ }^{26-34}$
Nevertheless, the logical design of new functional gels is difficult because of the lack of information about the molecular self-assembly process, many of the systems reported so far being examples of accidental discoveries. However, there are some general strategies to obtain a more rational design route toward new metallogels, which are based on either a molecular engineering approach or on structure-property correlations. ${ }^{35}$

A few years ago, we reported the preparation of $3 \mathrm{D}$ porous, crystalline metal-organic frameworks as well as stable viscoelastic metallogels, starting from the same organic ligand [i.e. 5-(1H-1,2,3-triazol-5-yl)isophthalic acid (5-TIA)], divalent metal ion (i.e. $\mathrm{Ca}(\mathrm{II}))$, and organic solvent [i.e. dimethylformamide (DMF)]. ${ }^{36}$ The system favored the formation of a crystalline MOF in the presence of water, whereas the water-free system induced gelation. A few years later, we demonstrated that the isosteric replacement, ${ }^{37,38}$ a

Received: November 26, 2018

Accepted: January 11, 2019

Published: January 28, 2019 
Scheme 1. Synthetic Procedure for the Preparation of Click-TIA

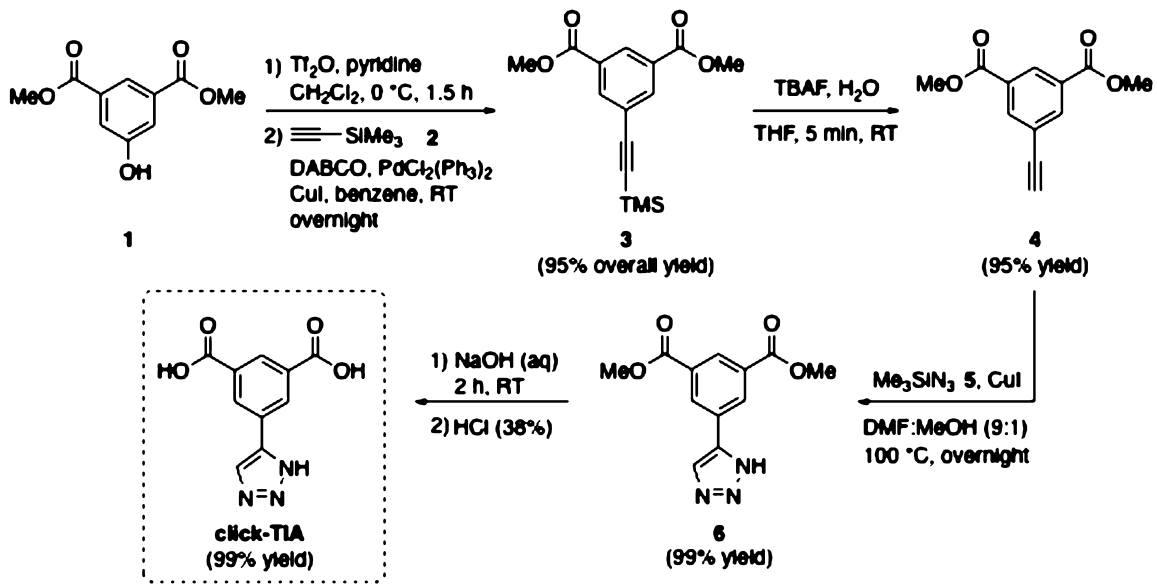

well-established paradigm in medicinal chemistry, could be successfully applied to the synthesis of new supramolecular gels with tailored functionality. ${ }^{39}$ Isosters ${ }^{40}$ have been defined as compounds or groups that possess near-equal molecular shapes and volumes, approximately the same distribution of electrons, and exhibit similar physical properties. ${ }^{41}$ As a first proof of concept, the gelation ability of a glutamic acid-based gelator was compared with its isostere, where the amide moiety was replaced by a 1,4-disubstituted 1,2,3-triazole unit. The results of that study indicated superior features in terms of gelation ability and gel properties for the materials prepared with the click-isosteric gelator in polar protic solvents, whereas the amide-based gelator was the best system for nonpolar solvents. $^{39}$ More recently, and in order to obtain new functional materials, we decided to apply the isosteric replacement on 5-TIA and we demonstrated that the simple substitution of the 1,2,4-triazole moiety by the $1 H-1,2,3$ triazol-5-yl unit enables the preparation of a new gelator (i.e. click-TIA) that can form hydrogels upon sonication, whereas 5-TIA alone lacks the ability to gel water. ${ }^{42}$

In this manuscript, we demonstrate that click-TIA can also be used to obtain new metallogels by combination with different metal acetates and sonication treatment. These metallogels have been characterized with different techniques, considering the gel prepared from click-TIA and copper(II) acetate as a model system.

\section{RESULTS AND DISCUSSION}

2.1. Synthesis and X-ray Crystal Structure of ClickTIA. Click-TIA was synthesized in four steps starting from commercially available dimethyl 5-hydroxyisophthalate (1) following our previously described procedure. ${ }^{42}$ Briefly, the alkyne unit was introduced into the aromatic ring by a classical Sonogashira cross-coupling reaction using trimethylsilylacetylene and the corresponding aromatic trifluoromethanesulfonate derivative of the starting dimethyl 5-hydroxyisophthalate (i.e. dimethyl 5-(((trifluoromethyl)sulfonyl)oxy)isophthalate) (Scheme 1). Deprotection of the trimethylsilyl group of the so-obtained alkyne $\mathbf{2}$ with tetra- $n$-butylammonium fluoride afforded dimethyl 5-ethynylisophthalate (3). Subsequent copper(I)-catalyzed azide-alkyne cycloaddition using azidotrimethylsilane as the azide source afforded the expected dimethyl 5-(1H-1,2,3-triazol-5-yl)isophthalate (4) in excellent overall yield (>95\%). Finally, alkaline hydrolysis of the ester groups yielded the desired click-TIA in nearly quantitative yield.

The X-ray crystal structure analysis of click-TIA further confirmed its chemical structure and solid-state packing. Single crystal of click-TIA was obtained from dimethyl sulfoxide (DMSO) solution by slow evaporation. From X-ray crystallography, it is evident that the asymmetric unit contains one molecule of click-TIA and one DMSO molecule. There is an $\mathrm{O}-\mathrm{H} \cdots \mathrm{O}$ hydrogen bond between click-TIA and DMSO molecule (Figure 1A). In higher order packing, the individual
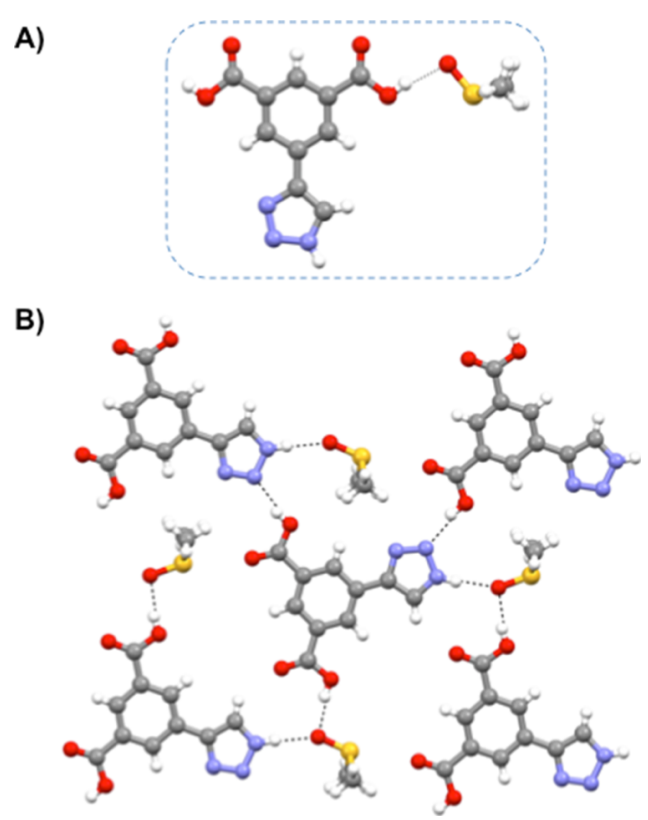

Figure 1. (A) Solid-state structure of click-TIA. The $\mathrm{O}-\mathrm{H} \cdots \mathrm{O}$ hydrogen bond is shown as dotted line. (B) Packing diagram of clickTIA showing DMSO-mediated supramolecular sheet-like structure. $\mathrm{O}-\mathrm{H} \cdots \mathrm{O}, \mathrm{N}-\mathrm{H} \cdots \mathrm{O}$, and $\mathrm{O}-\mathrm{H} \cdots \mathrm{N}$ hydrogen bonds are shown as dotted lines.

subunits of click-TIA are themselves regularly interlinked through multiple intermolecular hydrogen-bonding interaction, $\mathrm{O}-\mathrm{H} \cdots \mathrm{N}$ and DMSO-mediated hydrogen-bonding interactions and thereby form a supramolecular sheet-like structure along the crystallographic $b$ and $c$ directions (Figure 1B).

2.2. Preparation and Characterization of Metallogels. Control experiments showed that click-TIA alone does not 
form any gel in organic solvents, even after sonication. However, the combination of click-TIA with copper(II) acetate monohydrate $(\mathrm{CuA}), \mathrm{Cu}(\mathrm{OAc})_{2} \cdot \mathrm{H}_{2} \mathrm{O}$, and subsequent sonication resulted in the formation of blue opaque gels (Figure S1). Note that, only sonication or stirring is not enough to get a homogeneous gel. During initial experiments, we found that the use of other metal acetates also enabled the formation of metallogels under similar conditions (vide infra). At this point, we decided to take the mixture [click-TIA + $\mathrm{CuA}$ in DMF as a model system to optimize the gelation ability and characterize the corresponding metallogels. Thus, the critical gelation concentration (CGC) for this system was established as $0.1 \mathrm{M}$ in each component. Interestingly, different concentrations and ratios between click-TIA and $\mathrm{CuA}$ also afforded the formation of stable metallogels (Table 1).

Table 1. Gelation Ability of the Mixture [Click-TIA + CuA] in DMF at Different Concentrations and Metal/Ligand Ratios $^{a}$

$\begin{array}{ccccc}\text { entry } & \begin{array}{c}\text { concentration } \\ \text { click-TIA }(\mathrm{M})\end{array} & \begin{array}{c}\text { concentration } \\ \mathrm{CuA}(\mathrm{M})\end{array} & \begin{array}{c}\text { Ratio } \\ \text { click-TIA/CuA }\end{array} & \text { phase }^{b} \\ 1 & 0.1 & 0.1 & 1: 1 & \mathrm{G} \\ 2 & 0.1 & 0.2 & 1: 2 & \mathrm{PG} \\ 3 & 0.1 & 0.3 & 1: 3 & \mathrm{P} \\ 4 & 0.15 & 0.15 & 1: 1 & \mathrm{G} \\ 5 & 0.15 & 0.2 & 1: 1.3 & \mathrm{G} \\ 6 & 0.15 & 0.3 & 1: 2 & \mathrm{PG} \\ 7 & 0.2 & 0.075 & 2.7: 1 & \mathrm{~S} \\ 8 & 0.2 & 0.1 & 2: 1 & \mathrm{G} \\ 9 & 0.2 & 0.2 & 1: 1 & \mathrm{G} \\ 10 & 0.2 & 0.3 & 2: 3 & \mathrm{G} \\ 11 & 0.3 & 0.1 & 3: 1 & \mathrm{P} \\ 12 & 0.3 & 0.2 & 3: 2 & \mathrm{G} \\ 13 & 0.3 & 0.3 & 1: 1 & \mathrm{G} \\ 14 & 0.3 & 0.4 & 3: 4 & \mathrm{G} \\ 15 & 0.4 & 0.1 & 4: 1 & \mathrm{P} \\ 16 & 0.4 & 0.2 & 2: 1 & \mathrm{G} \\ 17 & 0.4 & 0.3 & 4: 3 & \mathrm{G} \\ 18 & 0.4 & 0.4 & 1: 1 & \mathrm{G}\end{array}$

${ }^{a}$ Upon sonication at RT for 5 min. ${ }^{b}$ Abbreviations: $\mathrm{G}=$ gel; PG $=$ partial gel; $\mathrm{P}=$ precipitate; $\mathrm{S}=$ solution. ${ }^{c}$ Gelation overnight. Note: unless otherwise indicated, gelation was observed immediately after sonication.

Moreover, a series of studies were carried out to determine the influence of the metal counter anion on the gelation phenomenon (Table S1). Interestingly, we found that only acetate anions are able to form gels in DMF. Only in the case of silver, triflate could be used as an anion to form a gel with click-TIA in toluene. Other anions such as nitrate, chlorate, fluoride, chloride, and sulfate led to the formation of clear solutions upon sonication at room temperature (RT). These results indicate that the counter anion plays an important role in the supramolecular assembly of $\mathrm{Cu}$ /click-TIA complexes.
Furthermore, the gelation ability of the gelator system [CuA + click-TIA] was evaluated for various solvents upon sonication at RT for $5 \mathrm{~min}$. We found that stable gels could be obtained immediately during sonication in at least three solvents: DMF, DMA, and pyridine. In contrast, partial gelation was observed in DMSO and glycerin (Table S2). $\mathrm{MeOH}$ was able to form a green gel after approximately one week. Interestingly, ethyl acetate and acetic anhydride decolorized the CuA solution, presumably through forming a $\mathrm{Cu}(\mathrm{I})$ species. Water afforded a clear solution of both the ligand and the metal salt, whereas the gelator system was found to be insoluble in the rest of the tested solvents (Table S1). It is important to emphasize that although click-TIA is able to form hydrogels in the absence of any metal salt, ${ }^{42}$ it is unable to gel organic solvents by its own. The effect of water to form either MOFs or metallogels was previously reported with 5TIA, ${ }^{36}$ the isostere of click-TIA. Therefore, we decided to check the role of water in the gelation ability of our model system [CuA (0.2 M) + click-TIA (0.2 M)] (Table S3). The results established a ratio $\mathrm{H}_{2} \mathrm{O} / \mathrm{DMF}$ of $2: 3(\mathrm{v} / \mathrm{v})$ as the maximum limit of water tolerance, above which neither gelation nor MOF formation was observed (i.e. only precipitate was obtained above this limit).

To gain additional insight into the complexation process, metallogels $1-3$ with $0.2 \mathrm{M}$ click-TIA and varying amounts of $\mathrm{CuA}(0.5-1.5$ equiv) were prepared and further investigated. Thus, metallogel-1 was prepared from the mixture click-TIA/ CuA 0.2 M:0.1 M, metallogel-2 from click-TIA/CuA 0.2 M:0.2 M, and metallogel-3 from click-TIA/CuA 0.2 M:0.3 M.

Oscillatory rheological experiments were performed in order to confirm the viscoelastic gel state and to examine the mechanical differences between metallogels 1-3 (Table 2 and Figure S2). First, the storage modulus $G^{\prime}$ and the loss modulus $G^{\prime \prime}$ were measured as a function of shear strain (dynamic strain sweep experiment, DSS) and angular frequency $\omega$ (dynamic frequency sweep experiment, DFS) to determine the linear viscoelastic regime. Within the linearity limits of deformation, $G^{\prime}$ was one order of magnitude higher than the loss modulus $G^{\prime \prime}$ in all three cases. Only for metallogel-1, a weak frequency dependence was observed. With increasing $\mathrm{CuA}$ content, the storage modulus was 13,960 , and $1810 \mathrm{~Pa}$ for metallogel-1, -2, and -3 , respectively. Interestingly, the highest dissipation factor, $\tan \delta\left(G^{\prime \prime} / G^{\prime}\right)$ value, was obtained for metallogel-1 $(\tan \delta=0.166)$ and the lowest for metallogel-2 $(\tan \delta=0.100)$ (Table 2). Moreover, the obtained critical stress or yield point $(\gamma)$ was significantly high for metallogel-2 with $80 \%$, whereas only 16 and $32 \%$ were achieved for metallogel-1 and -2 , respectively. These results suggest that metallogel-2 formed with an equimolar mixture of click-TIA and $\mathrm{CuA}$, which has the greatest mechanical stability and damping coefficient.

Fourier transform infrared (FT-IR) spectra of the xerogels obtained from the corresponding metallogels 1-3 (Figure S3) showed a strong absorption band of the carboxylic acid at 1640 $\mathrm{cm}^{-1}$, which lies between the frequencies of the free ligand at

Table 2. Rheological Properties of Metallogels-1, -2, and -3 Made of [CuA + Click-TIA $]^{a}$

\begin{tabular}{|c|c|c|c|c|c|c|}
\hline metallogel & concentration click-TIA (M) & concentration $\mathrm{CuA}(\mathrm{M})$ & $G^{\prime}(\mathrm{Pa})$ & $G^{\prime \prime}(\mathrm{Pa})$ & $\tan \delta$ & $\gamma(\%)$ \\
\hline 1 & 0.2 & 0.1 & 13 & 2 & $0.166 \pm 0.01$ & 16 \\
\hline 2 & 0.2 & 0.2 & 960 & 97 & $0.100 \pm 0.01$ & 80 \\
\hline 3 & 0.2 & 0.3 & 1810 & 212 & $0.112 \pm 0.01$ & 32 \\
\hline
\end{tabular}

${ }^{a}$ Rheological measurements were performed at $1 \mathrm{~Hz}$ frequency and $0.1 \%$ strain. 

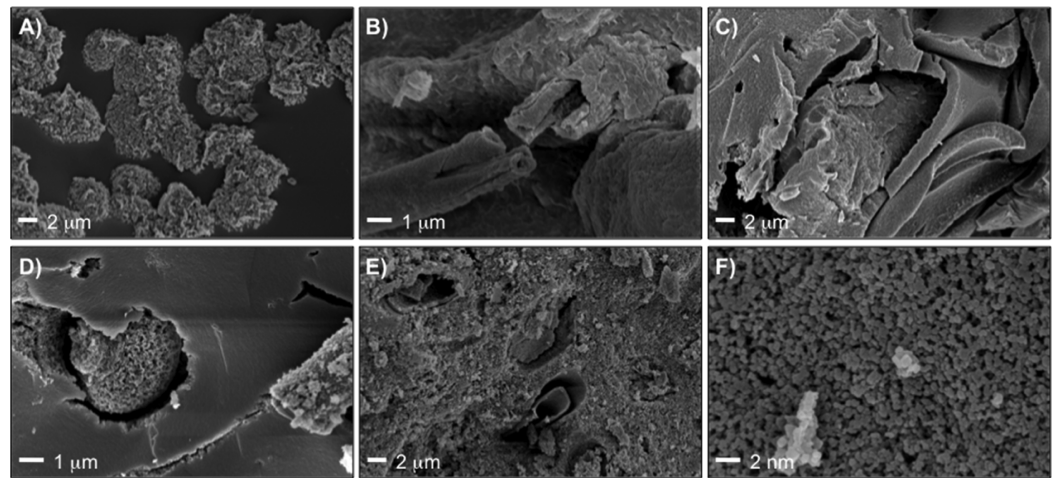

Figure 2. FE-SEM images of the xerogels of (A) metallogel-1, (B-D), metallogel-2, and (E,F) metallogel-3.

$1707 \mathrm{~cm}^{-1}$ and that from $\mathrm{CuA}$ at $1595 \mathrm{~cm}^{-1}$ because of metalcoordinated carboxylates. This is in agreement with the formation of strong metal-ligand complexes. Furthermore, free $\mathrm{CuA}$ typically shows two absorption bands at 1438 and $1420 \mathrm{~cm}^{-1}$, corresponding to antisymmetric $\nu_{\mathrm{a}}\left(\mathrm{COO}^{-}\right)$and symmetric versus $\left(\mathrm{COO}^{-}\right)$vibrations, whereas the xerogels displayed only one peak, which is shifted to lower frequencies $\left(1394 \mathrm{~cm}^{-1}\right)$. The spectroscopic analyses showed that the intensities of the peaks observed for the xerogels depend on the click-TIA/CuA ratio. Interestingly, the intensities decrease following the order: metallogel-2 > metallogel-1 > metallogel3 , that is, the xerogel prepared with the highest $\mathrm{CuA}$ content displays the lowest intensities. This can be explained considering that the increase of $\mathrm{Cu}^{2+}$ ion concentration facilitates more interactions with the carboxylic groups, leading to less free vibration of the $\mathrm{C}=\mathrm{O}$ stretching. Furthermore, this observation suggests that the orientation between click-TIA and $\mathrm{CuA}$ is different depending on the molar ligand/metal ratio.

The powder X-ray diffraction (PXRD) pattern of the xerogels obtained by freeze-drying (FD) the corresponding metallogels 1-3 was compared with that from pure click-TIA powder. The data revealed a structural evolution of ligand $/ \mathrm{Cu}$ aggregates with increasing $\mathrm{CuA}$ concentrations (Figure S4). Specifically, at 0.5 equiv, the pattern still resembles that of the pure ligand, although the reflexes at $2 \theta=21.9^{\circ}, 25.7^{\circ}$, and $27.4^{\circ}$ disappeared. At the equimolar ratio between click-TIA and $\mathrm{CuA}$, additional reflexes appeared at $2 \theta=2.2^{\circ}-19.9^{\circ}$, which is in concordance with an aggregation process. With further increase in $\mathrm{CuA}$ concentration to 1.5 equiv, only two reflexes at small $2 \theta\left(8.7^{\circ}\right.$ and $\left.10.4^{\circ}\right)$ were observed. The PXRD patterns showed that there are different types of assemblies between click-TIA and $\mathrm{CuA}$, which are ratiodependent and was further confirmed by field-emission scanning electron microscopy (FE-SEM) (vide infra).

The UV-vis spectrum of the ligand in DMF displayed a broad absorption at ca. $305 \mathrm{~nm}$ and a clear peak at ca. $266 \mathrm{~nm}$ responsible for $\pi-\pi^{*}$ transitions (Figure S5A). In contrast, pure $\mathrm{CuA}$ typically shows only one peak at ca. 280 with a tail extended until ca. $400 \mathrm{~nm}$. As expected, both the absorption bands and the fluorescence band (ca. $350 \mathrm{~nm}$ ) of the ligand increased with increasing concentration (Figure S5B). As expected, spectral changes were observed upon the addition of $\mathrm{CuA}$ aliquots to solutions of click-TIA (Figure S6). Interestingly, by increasing the amount of $\mathrm{CuA}$ the peak at ca. $305 \mathrm{~nm}$ disappeared and the peak at ca. $266 \mathrm{~nm}$ shifted to ca. $270 \mathrm{~nm}$, increasing until a ratio click-TIA/CuA 6:4 was reached. Further addition of $\mathrm{CuA}$ caused the decreasing of this peak again. The existence of nonideal isosbestic points suggested the formation of oligomers or polymers in the complexation process between click-TIA and $\mathrm{CuA}$. Thus, metal-ligand interactions developed between click-TIA and $\mathrm{CuA}$ provide not only thermodynamic stability but also kinetic liability to the metastable gel phase. Therefore, the competing thermodynamic pathway toward crystallization is slowed down with the help of additional noncovalent interactions, such as hydrophobic interactions, $\pi-\pi$ interactions, and hydrogen bonding. All these noncovalent interactions contribute to define the structure of the supramolecular gel, which is partly governed by the kinetics of phase separation and the viscosity of the system. On the basis of the X-ray analysis (vide supra), ligand-solvent interactions may also play a key role in the association and stabilization of the supramolecular aggregates involving multiple hydrogen bonds.

Additionally, field-emission scanning electron microscopy (FE-SEM) images of the xerogels obtained from the metallogels 1-3 were obtained in order to gain insights into the microstructure of the materials (Figures 2, S7-S9). The images confirmed that the $\mathrm{CuA}$ content remarkably influences the morphology of the xerogels. Specifically, the xerogel obtained from metallogel-1 composes spherical structures with sizes of ca. $5 \mu \mathrm{m}$ (Figure 1A), which consist of entangled fibers resembling hard corals with diameters below $0.5 \mu \mathrm{m}$ (Figure S7). Spherical structures were also observed with xerogels obtained from metallogel-2, that is, equimolar ratio between click-TIA and $\mathrm{CuA}$, although in this case, they seemed to be covered by a layer of ca. $2 \mu \mathrm{m}$ (Figure 1C,D). Besides spheres and layers, twined fibers were also observed in various specimens with a diameter of ca. $1-2 \mu \mathrm{m}$ and an inner cavity of ca. $200 \mathrm{~nm}$, which seems to be recoiled layers (Figure 1B). A porous material consisting of densed aggregates below 100 $\mathrm{nm}$ (Figure 1F) was observed with xerogels obtained from metallogel-3, which is interspersed with hollow tubes of ca. 4 $\mu \mathrm{m}$ (Figure 1E). Although, we have recorded representative images of the bulk materials to recognize any possible artifact, it should be stressed that significant changes in the microstructures could take place during the preparation of the dried samples and, hence, the interpretation of these images should always be done cautiously.

Interestingly, the metallogels displayed different thermostabilities at temperatures ranging from RT to $150{ }^{\circ} \mathrm{C}$, which was accompanied in all cases by a color change from greenishblue to green (Figure S10). The highest thermostability was observed for metallogel-1, whereas metallogel-2 lost about half of the liquid phase during the ramp of temperature. In addition, metallogel-2 and metallogel-3 seemed to get 
inhomogeneous after heating to $150{ }^{\circ} \mathrm{C}$, which needs to be further investigated in order to draw mechanistic conclusions. It is worth mentioning that heating the metallogels for prolonged time caused the formation of precipitates instead of gel-to-sol transitions. The specific nature of these precipitates (presumably metal organic frameworks) will be evaluated in future research.

Finally, preliminary experiments demonstrated that clickTIA is also able to form stable fibrillar gels with a variety of other metal salts such as $\mathrm{Pd}(\mathrm{OAc})_{2}$ (brown gel), $\mathrm{Zn}(\mathrm{OAc})_{2}$ (ivory-colored gel), and $\mathrm{Mn}(\mathrm{OAc})_{3} \bullet 4 \mathrm{H}_{2} \mathrm{O}$ (dark brown gel) (Figures S11-S13). These gels were obtained at RT both in DMF and DMSO, following the same general procedure used for the preparation of the gel made using copper acetate (vide supra). Initial UV-vis and fluorescence measurements confirmed the formation of metal complexes (Figure S12). Further studies of these and other potential metallogels based on click-TIA are currently underway in our laboratories and the results will be reported in due course.

\section{CONCLUSIONS}

In conclusion, the compound 5-TIA (click-TIA) constitutes a versatile ligand for the preparation of new metallogels upon controlled combination with different metal acetates. The results obtained from the study of the model system formed by the mixture click-TIA and copper(II) acetate have revealed a series of critical factors that dominate the supramolecular assembly of the metal complexes. Thus, sonication of the initial mixture, ligand/metal ratio, nature of the solvent, and type of the counter anion play critical roles in the formation of stable viscoelastic $3 \mathrm{D}$ networks with specific morphologies, thermal, and mechanical properties.

\section{EXPERIMENTAL SECTION}

4.1. Materials and Methods. Unless otherwise noted, all reagents were purchased from commercial suppliers and used as received without further purification. All solvents for synthesis were dried by distillation. Compound 5-TIA (clickTIA) was synthesized and purified, following our previously reported procedure. ${ }^{42}{ }^{1} \mathrm{H}$ NMR (500 MHz, DMSO- $\left.d_{6}\right): \delta$ (ppm) 8.43 (s, 1H), 8.63 (s, 3H), 13.44 (br s, 2H), 15.26 (br s, $1 \mathrm{H}) ;{ }^{13} \mathrm{C}$ NMR (125 MHz, DMSO- $\left.d_{6}\right): \delta(\mathrm{ppm}) 129.3(\mathrm{~d})$, 130.0 (d), 132.2 (s), 166.5 (s); mp 271-272 ${ }^{\circ} \mathrm{C}$; HRMS (ESI): $m / z$ calcd for $\mathrm{C}_{10} \mathrm{H}_{6} \mathrm{~N}_{3} \mathrm{O}_{4}[\mathrm{M}-\mathrm{H}], 232.0358$; found, 232.0357. Sonication treatments were done using a sonication bath USC200th VWR, $45 \mathrm{kHz}, 60 \mathrm{~W}$. UV-vis spectra were obtained on Ocean Optics DH2000-BAL UV-vis/NIR or on a PerkinElmer (Lambda 35) spectrophotometer using a $1 \mathrm{~cm}$ path length quartz cell. FT-IR spectra were obtained with an Agilent Technologies Cary 630 FTIR spectrometer equipped with Golden Gate Diamond ATR (attenuated total reflection). Fluorescence spectra were obtained on a PerkinElmer fluorescent spectrometer (LS 55) using a $1 \mathrm{~cm}$ path length quartz cell. Slit widths 2.5/2.5 were used. Single crystal X-ray diffraction experiment was performed on a Bruker APEX-2 CCD diffractometer with Mo $\mathrm{K} \alpha$ radiation. Data were processed using the Bruker SAINT package and the structure solution and refinement procedures were performed using SHELX97. The nonhydrogen atoms were refined with anisotropic thermal parameters. Crystallographic data of click-TIA: $\mathrm{C}_{10} \mathrm{H}_{7} \mathrm{~N}_{3} \mathrm{O}_{4}, \mathrm{C}_{2} \mathrm{H}_{6} \mathrm{O}_{6}, M_{\mathrm{w}}=311.31$, space group $P 121 / N 1, a=6.7758(7), b=15.4192(11), c=13.0681(11) \AA$, $\alpha=6.7758^{\circ} \beta=95.261^{\circ} \gamma=90^{\circ}, \mathrm{V}=1359.6(2) \AA 33, Z=4, \mathrm{dm}$ $=1.521 \mathrm{Mgm}^{-3}, T=100 \mathrm{~K}, R_{1} 0.1427$ and $\mathrm{wR}_{2} 0.3550$ for 2274 data with $I>2 \sigma(I)$. The data for click-TIA have been submitted at the Cambridge Crystallographic Data Center with reference number CCDC 1881063. PXRD experiments were performed on a STOE STADI P powder diffractometer (Start Fragment Transmission mode, flat samples, Dectris Mythen $1 \mathrm{k}$ microstrip solid-state detector, Debye-Scherrer geometry) with $\mathrm{Cu} \mathrm{K} \alpha 1$ radiation $(\lambda=1.540598 \AA)$ operated at $40 \mathrm{kV}$ and $40 \mathrm{~mA}$. The experiments were measured in the range $2^{\circ} \leq$ $2 \theta \leq 90^{\circ}$. Conditions: start fragment $0.0151^{\circ}$, time $60 \mathrm{~s} / \mathrm{step}$. Lattice spacings were calculated from Bragg's law. CGC, defined as the minimum gelator concentration (both click-TIA and metal salt) necessary for gelation, was estimated by weighting different amounts of click-TIA and adding different amounts of the previously prepared metal acetate stock solution, and applying the sonication protocol as described above. Herein, CGC was established with respect to the absence of gravitational flow. Dynamic oscillatory rheological measurements of the gels (total volume $=2 \mathrm{~mL}$ ) were performed with an AR 2000 Advanced rheometer (TA Instruments) equipped with a Julabo $\mathrm{C}$ cooling system. A $500 \mu \mathrm{m}$ gap setting and a torque setting of $5 \times 10^{-4} \mathrm{~N} / \mathrm{m}$ at 25 ${ }^{\circ} \mathrm{C}$ were used for the measurements in a plain-plate geometry ( $40 \mathrm{~mm}$, stainless steel). Evolution of the storage $\left(G^{\prime}\right)$ and loss $\left(G^{\prime \prime}\right)$ moduli was studied as follows. DSS measurements were first performed between 0.1 and $100 \%$ strain at $1 \mathrm{~Hz}$ frequency to establish the strain value at which reasonable torque values were given (ca. 10 times of the transducer resolution limit). Subsequently, DFS measurements (from 0.1 to $10 \mathrm{~Hz}$ at $0.1 \%$ strain) and time sweep measurements (DTS) within the linear viscoelastic regime ( $0.1 \%$ strain and $1 \mathrm{~Hz}$ frequency) were carried out. Field-Emission Scanning Electron Microscopy (FE-SEM) images of the bulk xerogels were obtained with Zeiss Merlin, FE-SEM (accelerating voltage $10 \mathrm{kV}$ ) or with a JEOL scanning microscope-JSM-6700F (accelerating voltage $5-7 \mathrm{kV}$ ). For visualization, samples were prepared by the FD method as following: A $4 \mathrm{~mL}$ glass vial containing the corresponding gel (total volume $=1 \mathrm{~mL}$ ) was frozen in liquid nitrogen and the solvent was immediately evaporated under reduced pressure $(0.6 \mathrm{mmHg})$ overnight at RT. The obtained fibrous solid was placed on top of a tin plate and shielded with $\mathrm{Au}$ or Pt (40 mA during 30-60 s; film thickness $=5-10 \mathrm{~nm})$. Images were taken at the University of Zaragoza (Servicio General de Apoyo a la Investigación-SAI) and at the IISER Kolkata FE-SEM facility.

4.2. General Procedure for the Preparation of Metallogels. Typically, a weighted amount of click-TIA and an appropriate amount of a metal acetate stock solution were placed into a screw-capped glass vial $(4 \mathrm{~cm}$ length $\times 1 \mathrm{~cm}$ diameter). The mixture was quickly stirred with a spatula and then sonicated for ca. $5 \mathrm{~min}$ at RT until everything was homogeneous. The resulting solution was maintained at RT until the gel was formed. The material was preliminary classified as the gel if it did not exhibit gravitational flow upon turning the vial upside-down at RT to form a green-blue homogeneous gel. The gel state was further confirmed by oscillatory rheological measurements. Three model formulations with different click-TIA/CuA ratios were used for further characterization: metallogel-1 (click-TIA/CuA 0.2 M:0.1 M), metallogel-2 (click-TIA/CuA 0.2 M:0.2 M), and metallogel-3 (click-TIA/CuA $0.2 \mathrm{M}: 0.3 \mathrm{M}$ ). The same general procedure 
was used to prepare metallogels using other metal salts as indicated in the main text.

\section{ASSOCIATED CONTENT}

\section{S Supporting Information}

The Supporting Information is available free of charge on the ACS Publications website at DOI: 10.1021/acsomega.8b03292.

Solvent screening, role of metal and water in gel formation, rheological measurements, X-ray data, spectroscopic data, thermostability studies, and additional figures and tables (PDF)

\section{AUTHOR INFORMATION}

\section{Corresponding Author}

*E-mail: David.Diaz@chemie.uni-regensburg.de, d.diaz.diaz@ ipna.csic.es.

\section{ORCID}

Víctor S. Martín: 0000-0003-0300-9636

David Díaz Díaz: 0000-0002-0557-3364

\section{Author Contributions}

The manuscript was written through contributions of all authors. All authors have given approval to the final version of the manuscript.

\section{Funding}

Deutsche Forschungsgemeinschaft (DFG 1748/3-1; 1748/32), Universität Regensburg, Spanish MINECO, cofinanced by the European Regional Development Fund (ERDF) (CTQ214-56362-C2-1-P) and Fondecyt Iniciacion (11160707).

\section{Notes}

The authors declare no competing financial interest.

\section{ACKNOWLEDGMENTS}

Financial from Deutsche Forschungsgemeinschaft (DFG 1748/3-1; 1748/3-2), Universität Regensburg, Spanish MINE$\mathrm{CO}$, cofinanced by the European Regional Development Fund (ERDF) (CTQ214-56362-C2-1-P), and Fondecyt Iniciacion (11160707) are gratefully acknowledged. S.K.N. thanks CSIR, India for Fellowship. D.H. thanks IISER Kolkata for FE-SEM facility, and Prof. Dr. A. Göpferich and his group (Universität Regensburg) for assistance with rheological measurements. D.D.D. thanks DFG for the Heisenberg Professorship Award.

\section{REFERENCES}

(1) Huang, Y.; Ding, Y.; Li, T.; Yang, M. Redox Hydrogel Based Immunosensing Platform for the Label-Free Detection of a Cancer Biomarker. Anal. Methods 2015, 7, 411-415.

(2) Drury, J. L.; Mooney, D. J. Hydrogels for Tissue Engineering: Scaffold Design Variables and Applications. Biomaterials 2003, 24, 4337-4351.

(3) Ellis-Behnke, R. G.; Liang, Y.-X.; You, S.-W.; Tay, D. K. C.; Zhang, S.; So, K.-F.; Schneider, G. E. Nano Neuro Knitting: Peptide Nanofiber Scaffold for Brain Repair and Axon Regeneration with Functional Return of Vision. Proc. Natl. Acad. Sci. U.S.A. 2006, 103, 5054-5059.

(4) Tibbitt, M. W.; Anseth, K. S. Hydrogels as Extracellular Matrix Mimics for 3D Cell Culture. Biotechnol. Bioeng. 2009, 103, 655-663.

(5) Qu, F.; Zhang, Y.; Rasooly, A.; Yang, M. Electrochemical Biosensing Platform Using Hydrogel Prepared From Ferrocene Modified Amino Acid as Highly Efficient Immobilization Matrix. Anal. Chem. 2014, 86, 973-976.
(6) Jhaveri, S. J.; McMullen, J. D.; Sijbesma, R.; Tan, L.-S.; Zipfel, W.; Ober, C. K. Direct Three-Dimensional Microfabrication of Hydrogels Via Two-Photon Lithography in Aqueous Solution. Chem. Mater. 2009, 21, 2003-2006.

(7) Díaz, D. D.; Kühbeck, D.; Koopmans, R. J. Stimuli-Responsive Gels as Reaction Vessels and Reusable Catalysts. Chem. Soc. Rev. 2011, 40, 427-448.

(8) Kiyonaka, S.; Sugiyasu, K.; Shinkai, S.; Hamachi, I. First Thermally Responsive Supramolecular Polymer Based on Glycosylated Amino Acid. J. Am. Chem. Soc. 2002, 124, 10954-10955.

(9) Adhikari, B.; Palui, G.; Banerjee, A. Self-Assembling Tripeptide Based Hydrogels and Their Use in Removal of Dyes From WasteWater. Soft Matter 2009, 5, 3452-3460.

(10) Chakraborty, P.; Roy, B.; Bairi, P.; Nandi, A. K. Improved Mechanical and Photophysical Properties of Chitosan Incorporated Folic Acid Gel Possessing the Characteristics of Dye and Metal Ion Absorption. J. Mater. Chem. 2012, 22, 20291-20298.

(11) Bera, S.; Haldar, D. A Rechargeable Self-Healing Safety Fuel Gel. J. Mater. Chem. A 2016, 4, 6933-6939.

(12) Kiyonaka, S.; Sada, K.; Yoshimura, I.; Shinkai, S.; Kato, N.; Hamachi, I. Semi-Wet Peptide/Protein Array Using Supramolecular Hydrogel. Nat. Mater. 2003, 3, 58-64.

(13) Vemula, P. K.; John, G. Smart Amphiphiles: Hydro/ Organogelators for in situ Reduction of Gold. Chem. Commun. 2006, 2218-2220.

(14) Estroff, L. A.; Hamilton, A. D. Water Gelation by Small Organic Molecules. Chem. Rev. 2004, 104, 1201-1218.

(15) Smith, D. K. Building bridges. Nat. Chem. 2010, 2, 162-163.

(16) Sangeetha, N. M.; Maitra, U. Supramolecular Gels: Functions and Uses. Chem. Soc. Rev. 2005, 34, 821-836.

(17) Ajayaghosh, A.; Praveen, V. K.; Vijayakumar, C. Organogels as Scaffolds for Excitation Energy Transfer and Light Harvesting. Chem. Soc. Rev. 2008, 37, 109-122.

(18) George, M.; Weiss, R. G. Molecular Organogels. Soft Matter Comprised of Low-Molecular-Mass Organic Gelators and Organic Liquids †. Acc. Chem. Res. 2006, 39, 489-497.

(19) Samai, S.; Dey, J.; Biradha, K. Amino Acid Based LowMolecular-Weight Tris(bis-amido) Organogelators. Soft Matter 2011, 7, 2121-2126.

(20) Jones, C. D.; Tan, J. C.; Lloyd, G. O. Supramolecular Isomerism of a Metallocyclic Dipyridyldiamide Ligand Metal Halide System Generating Isostructural $(\mathrm{Hg}, \mathrm{Co}$ and $\mathrm{Zn})$ Porous Materials. Chem. Commun. 2012, 48, 2110-2112.

(21) Le Bideau, J.; Viau, L.; Vioux, A. Ionogels, Ionic Liquid Based Hybrid Materials. Chem. Soc. Rev. 2011, 40, 907-925.

(22) Yu, X.; Chen, L.; Zhang, M.; Yi, T. Low-Molecular-Mass Gels Responding to Ultrasound and Mechanical Stress: Towards SelfHealing Materials. Chem. Soc. Rev. 2014, 43, 5346-5371.

(23) Dastidar, P. Supramolecular Gelling Agents: Can They Be Designed? Chem. Soc. Rev. 2008, 37, 2699-2715.

(24) Ray, S.; Das, A. K.; Banerjee, A. pH-Responsive, Bolaamphiphile-Based Smart Metallo-Hydrogels as Potential Dye-Adsorbing Agents, Water Purifier, and Vitamin B12Carrier. Chem. Mater. 2007, 19, 1633-1639.

(25) Pal, A.; Basit, H.; Sen, S.; Aswal, V. K.; Bhattacharya, S. Structure and Properties of Two Component Hydrogels Comprising Lithocholic Acid and Organic Amines. J. Mater. Chem. 2009, 19, 4325.

(26) Fages, F. Metal Coordination to Assist Molecular Gelation. Angew. Chem., Int. Ed. 2006, 45, 1680-1682.

(27) Piepenbrock, M.-O. M.; Lloyd, G. O.; Clarke, N.; Steed, J. W. Metal- and Anion-Binding Supramolecular Gels. Chem. Rev. 2010, 110, 1960-2004.

(28) Filby, M. H.; Steed, J. W. A Modular Approach to Organic, Coordination Complex and Polymer Based Podand Hosts for Anions. Coord. Chem. Rev. 2006, 250, 3200-3218.

(29) Zhang, J.; Su, C.-Y. Metal-Organic Gels: From Discrete Metallogelators to Coordination Polymers. Coord. Chem. Rev. 2013, $257,1373-1408$. 
(30) Murata, K.; Aoki, M.; Nishi, T.; Ikeda, A.; Shinkai, S. New Cholesterol-Based Gelators with Light- and Metal-Responsive Functions. J. Chem. Soc., Chem. Commun. 1991, 1715-1718.

(31) Tam, A. Y.-Y.; Yam, V. W.-W. Recent Advances in Metallogels. Chem. Soc. Rev. 2013, 42, 1540-1567.

(32) Murata, K.; Aoki, M.; Suzuki, T.; Harada, T.; Kawabata, H.; Komori, T.; Ohseto, F.; Ueda, K.; Shinkai, S. Thermal and Light Control of the Sol-Gel Phase Transition in Cholesterol-Based Organic Gels. Novel Helical Aggregation Modes as Detected by Circular Dichroism and Electron Microscopic Observation. J. Am. Chem. Soc. 1994, 116, 6664-6676.

(33) Häring, M.; Díaz, D. D. Supramolecular Metallogels with Bulk Self-Healing Properties Prepared by in situ Metal Complexation. Chem. Commun. 2016, 52, 13068-13081.

(34) Westcott, A.; Sumby, C. J.; Walshaw, R. D.; Hardie, M. J. Metallo-Gels and Organo-Gels with Tripodal CyclotriveratryleneType and 1,3,5-Substituted Benzene-Type Ligands. New J. Chem. 2009, 33, 902-912.

(35) Dastidar, P.; Roy, R.; Parveen, R.; Ganguly, S.; Majumder, J.; Paul, M. Functional Supramolecular Materials: From Surfaces to MOFs, Banerjee, R., Ed.; RSC, 2017, chapter 2.

(36) Mallick, A.; Schön, E.-M.; Panda, T.; Sreenivas, K.; Díaz, D. D.; Banerjee, R. Fine-tuning the balance between crystallization and gelation and enhancement of $\mathrm{CO} 2$ uptake on functionalized calcium based MOFs and metallogels. J. Mater. Chem. 2012, 22, 1495114963.

(37) Brown, N. Bioisosteres in Medicinal Chemistry; Wiley-VCH: Weinheim, 2012; Vol. 54.

(38) Hamada, Y.; Kiso, Y. The Application of Bioisosteres in Drug Design for Novel Drug Discovery: Focusing on Acid Protease Inhibitors. Expert Opin. Drug Discovery 2012, 7, 903-922.

(39) Bachl, J.; Mayr, J.; Sayago, F. J.; Cativiela, C.; Díaz, D. D. Amide-Triazole Isosteric Substitution for Tuning Self-Assembly and Incorporating New Functions into Soft Supramolecular Materials. Chem. Commun. 2015, 51, 5294-5297.

(40) Thornber, C. W. Isosterism and Molecular Modification in Drug Design. Chem. Soc. Rev. 1979, 8, 563-580.

(41) Burger, A. Isosterism and Bioisosterism in Drug Design. Progress in Drug Research; Springer, 1991; Vol. 37, pp 287-371.

(42) Häring, M.; Rodríguez-López, J.; Grijalvo, S.; Tautz, M.; Eritja, R.; Martín, V. S.; Díaz Díaz, D. Isosteric Substitution of 4H-1,2,4Triazole by $1 H-1,2,3$-Triazole in Isophthalic Derivative Enabled Hydrogel Formation for Controlled Drug Delivery. Mol. Pharm. 2018, 15, 2963-2972. 